\title{
Dynamics of a vibration system, taking into account the hereditary type friction and the mobility of a vibration limiter
}

\author{
Vladimir S. Metrikin*, Leonid A. Igumnov, Mikhail V. Zaitsev, Aleksandr V. Boev \\ Research Institute for Mechanics, National Research Lobachevsky State University of Nizhny \\ Novgorod, Nizhny Novgorod, Russian Federation
}

\begin{abstract}
The paper investigates the dynamics of some vibration systems taking into account the hereditary-type dry friction and mobility of a vibration limiter. The interaction of the vibration limiter and the vibration system occurs either according to Newton's hypothesis (an absolutely rigid limiter) or softly (the limiter is mobile). A general mathematical model (MM) of systems has been developed, which is highly non-linear nonautonomous system with variable structure. A numerical-analytical approach using the mathematical apparatus of the point mapping method is implemented to study the dynamics of the MM. The peculiarity in the research approach is that the point mapping is not formed by the classical method (mappings of the Poincaré surface into itself), but by the duration of relative rest of the vibration system, which greatly facilitated the process of point mapping and its detailed study. The presence of floating boundaries of slip-motion plates required the creation of an original approach in constructing point mapping and interpreting the results obtained. The structure of the phase portrait of the MM as a function of the characteristics of the sliding and state friction forces, as well as on the type and position of the limiter was studied using the developed research methodology and the created software product. Based on the character of changes in bifurcation diagrams, it was possible to determine the main laws of changes in the motion regimes (occurrence of random complexity via the period possible transfer to chaos doubling process) when changing the parameters of the vibration system (amplitude and frequency of periodic action, the form of the functional dependence describing the change in the coefficient of friction of relative rest. Analytical results with and without a vibration limiter are compared.
\end{abstract}

\section{Introduction}

The problem of modeling and investigation of dynamic systems taking into account dry friction remains still relevant due to significant difficulties of adequate mathematical description of the process in real objects. It is proved in the early works by A.Yu. Ishlinsky and I.V. Kragelsky on stick-slips at friction [1], works by N.V. Butenina on "stick-slip"

\footnotetext{
* Corresponding author: v.s.metrikin@mail.ru
} 
hypothesis [2], the results of which have found wide application and development at present [3-12]. Thus, the paper [9] is devoted to the study of the dynamics of the drilling rig, the physical model of which is presented in the form that coincides with the model in works [3, 4] for a number of possible drilling regimes and can be adapted to the present model. The work [10] reviews the bifurcation phenomena related to non-smooth dynamical systems by an example of two models, one of which is similar to the model presented in works [3, 4] (without considering the hereditary-type friction), the other one is the "woodpecker" with impacts. A simple and effective alternative friction model for sticking simulation at vibrations is presented in [6]. The mathematical model of alternative friction consists of a set of ordinary non-rigid differential equations and has the advantage that it can be integrated with any standard ODE-solver. Comparison to the smoothing method $[10,11]$ proves that the alternative friction model is more effective in terms of computation. However, not much is known on bifurcations of discontinuous vector fields. Discontinuous dynamical systems arise due to physical discontinuities, such as dry friction, impact and clearance in mechanical systems or diode elements in electrical circuits.

The results of study of frictional self-oscillations in a single-mass and double-mass systems accounting for visco-dry friction in the relative motion of solids (without considering the hereditary- type friction) are presented in [12]. The dynamics of a vibratory system taking into account dry friction of the hereditary- type and the vibration limiter are being studied in the present paper. The vibration limiter and the vibration system interact either according to Newton's hypothesis or taking into account the elasticity of the wall of the limiter. A mathematical model (MM) which is a strongly nonlinear non-autonomous system with a variable structure has been developed. The dynamics of the MM is analyzed numerically and analytically, using the mathematical apparatus of the point mapping method. Employing the newly-developed software product and the original investigation methodology, the structure of the phase portrait of the MM has been analyzed as a function of the characteristics of friction forces of sliding and rest, as well as of the type and location of the limiter. Based on the variation character of bifurcation diagrams, it was possible to find the main laws of the process of motion mode alterations with the changing parameters of the oscillation system.

\section{Mathematical models}

A.Yu. Ishlinsky and I.V. Kragelsky in [1] introduced a hypothesis that a coefficient of friction of relative rest (CFRR) of two sliding contacting bodies is not constant, but depends on their "previous" time duration of sliding with identical speeds. After a considerable delay, the hypothesis gained attention of scientists, engaged in research of friction systems (see [2-4] and the related references). In these papers, a number of new results are cited on the example of the simplest nonlinear autonomous dynamical systems. It has been shown that, in comparison with the known studies of the systems with a constant CFRR, in systems with variable CFRR there exist random complex periodic and stochastic motion regimes. Dynamic vibration systems, whose scheme coincides with the one studied in [2-4], were also considered by foreign scientists (see [5-7] and the related references), but the hypothesis of A.Yu. Ishlinsky and I.V. Kragelsky was not employed. The classical model of static friction was used in those works. The physical system used as a prototype for constructing the mathematical model is a body of mass $\mathrm{m}$ located on a rough belt moving at a constant velocity $\mathrm{V}$. 


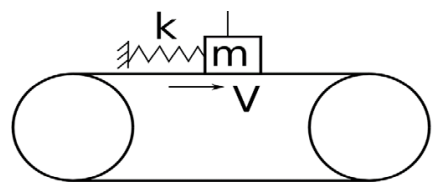

a

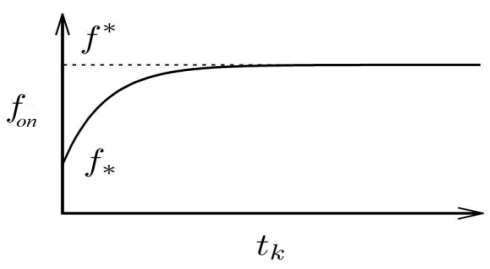

b

Fig. 1. Physical model of the system

The load is fixed by a spring with stiffness coefficient $k$ to the fixed support Fig. 1a. The load is affected by the frictional force and, possibly, the periodic external force $f(t)$ (example: drilling of deep wells [9]). The movement of the load in the direction of the belt movement is limited by the wall located at a distance «a» from the equilibrium position of the load with a fixed belt. Systems of this type, with small variations, are well known and many of their variations have been well studied. It is known that the simplest system of this kind, without external force and a wall, has one stable limit cycle in its phase space. Variations of such a system with alternative friction models of relative rest were not much covered in the scientific literature. In this work it is assumed that the sliding friction coefficient $f_{*}$ is constant, and the coefficient of friction of the relative rest, according to the hypothesis of A.Yu. Ishlinsky and I.V. Kragelski [1], is a continuous monotonically increasing function of time tk of a long-term contact (coincidence of mass velocities and the belt) of these bodies Fig. 1b. Coulomb-Amonton friction was chosen as a mathematical model of frictional forces. The impact on the wall is assumed to be instantaneous, with a velocity recovery coefficient $R$.

A mathematical model of the system considered can be written as

$$
\begin{gathered}
m \ddot{x}+k x=f(t)-f_{*} P \operatorname{sign}(\dot{x}-V(t)), \dot{x} \neq V(t), x<a, \\
|k x-f(t)+m \dot{V}(t)| \leq f_{\text {on }}\left(t_{k}\right) P, \dot{x}=V(t), x<a, \\
\dot{x}^{+}=-R \dot{x}^{-}, x=a, \dot{x}^{-}>0,
\end{gathered}
$$

where the first equation describes the law of motion of the body, accounting for sliding friction coefficient $f_{*}$, with a velocity different from the velocity of the belt; the second inequality defines a relation of the forces at which, if it is satisfied, the body moves with the velocity equal to that of the belt with the account of the form of CFRR $-f_{\text {on }}\left(\tau_{k}\right)$ (fig. 1b). The third equation describes a model of the impact of the load against a wall. Introducing dimensionless time $\tau=\omega t$, variable $\xi=k x / f_{*} P$ and parameters $\theta=V(t) \sqrt{\mathrm{km}} / f_{*} P$, system (1)-(3) can be rewritten as

$$
\begin{gathered}
\ddot{\xi}+\xi+\operatorname{sign}(\dot{\xi}-\theta)=F(\tau), \dot{\xi} \neq \theta, \xi<b, \\
\ddot{\xi}+\xi+\operatorname{sign}(\dot{\xi}-\theta)=F(\tau), \dot{\xi} \neq \theta, \xi<b, \\
\dot{\xi}^{+}=-R \xi^{-}, \xi=b, \dot{\xi}^{-}>0,
\end{gathered}
$$

where $b=k a / f_{*} P, \rho=c R / f_{*} P, \varepsilon(\tau)=\left(f_{\text {on }}(\tau)-f_{*}\right) / f_{*}, \varepsilon_{k}=\varepsilon\left(\tau_{k}\right)$. 


\section{Structure of the phase space}

Since the system is non-autonomous and can be described by a second-order differential equation with a variable structure, its state is a triplet $\{\xi, \dot{\xi}, \tau\}$, and its phase space is threedimensional, respectively. Trajectories in it can exist only in a half-space $\xi \leq b$. It can be seen, that there is a plate of sliding motions in the plane $\Pi[5](-1 \leq \xi \leq+1, \dot{\xi}=\theta)$. If the image point hits $\Pi_{c}$, its movement will change according to the law $\xi=\theta \tau+\xi_{P}, \dot{\xi}=\theta$, where $\xi_{P} \in \Pi_{c}$. The dimensionless functional dependence of the CFRR $\varepsilon\left(\tau_{k}\right)$ on time $\tau_{k}$ of prolonged contact was taken in the form

$$
\varepsilon\left(\tau_{k}\right)=\left\{\begin{array}{c}
\varepsilon \tau_{k}, \tau_{k}<\varepsilon_{*} \\
\varepsilon_{*}, \tau_{k} \geq \varepsilon_{*}
\end{array}\right.
$$

Since the depicting point will always end on the plate of sliding movements, the dynamics of the system can be investigated by studying the properties of the point mapping of the half-line $L(-1 \leq \xi, \dot{\xi}=\theta)$ into itself, or the properties of numerical sequence, elements of which are equal to durations $\tau_{k}, k=1,2,3 \ldots$. To study the dynamics of the system using the sequence function, a software product on the Java platform was developed, allowing to make calculation for various values of the system parameters and the construction of phase trajectories in three-dimensional phase space, the explicit form of the succession function and bifurcation diagrams.

Thus, Fig. 2 (a, c) depicts phase portraits, Fig. 2 (b, d) shows the corresponding Lamereya diagrams for different values of the velocity recovery factor for the impact, and Fig. 3 demonstrates bifurcation diagram using the method of symbolic dynamics (lines of corresponding color are responsible for a certain periodic regime of motion of the system, given by the corresponding set of letters, given below).

a)
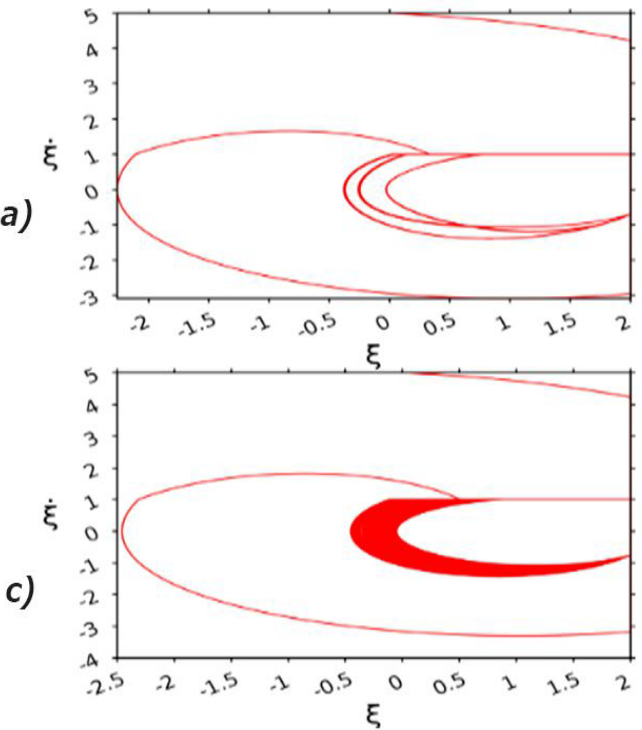
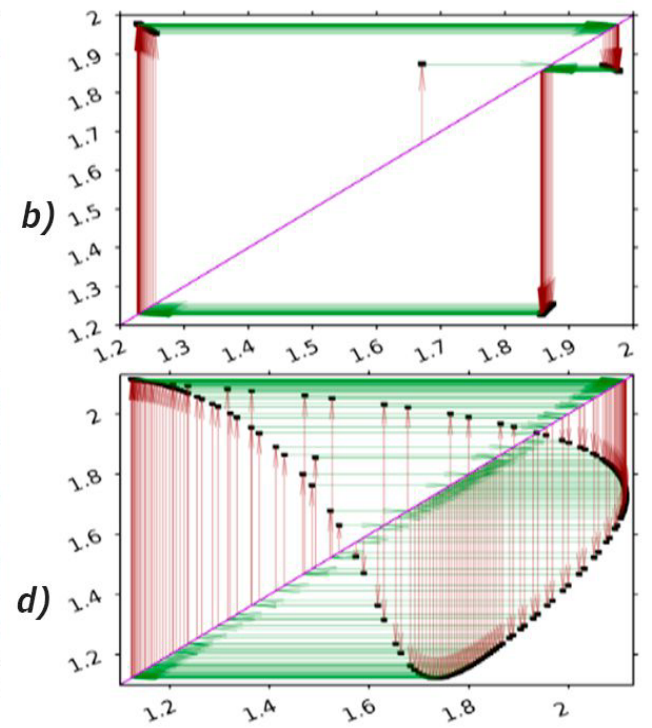

Fig. 2. Bifurcation diagrams and corresponding Lamereya diagrams. 


\section{Bifurcation diagram}

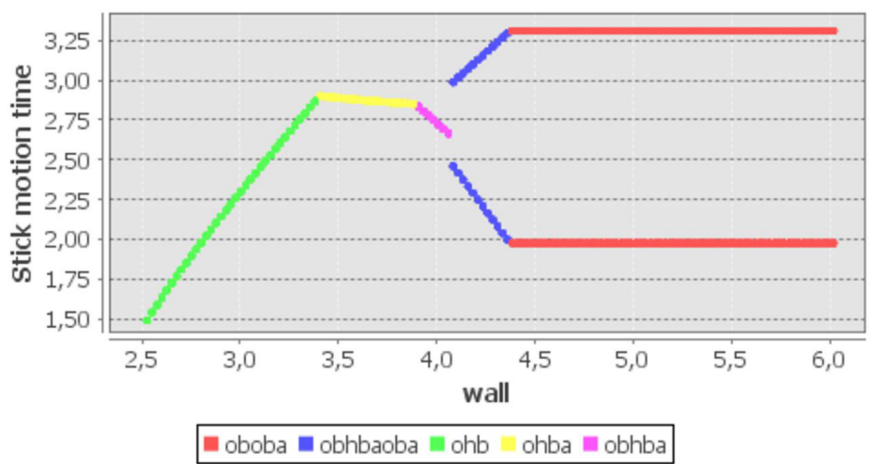

Fig. 3. Bifurcation diagram

It is evident from the pictures that for $R=0.7$ (Fig. 2a) the system has a stable limiting cycle describing frictional oscillations of the body with three prolonged stops symbolically represented as "ohbohbohb", meaning that the first stop "o" is followed by an impact against the wall "h" and then segment "b" in half-space $\dot{\xi}<\theta$, which is followed by two more similar circles "ohb", and then the cycle is repeated. For $R=0.75$ (Fig. 2c) the behavior of the system is chaotic.

Fig. 3 represents a bifurcation diagram describing the various complex periodic motions of the system when the location of the obstacle is changed (the character of periodic motion is recognized by a sequence of symbols). Fig. 4 presents more complex dynamics, illustrating transfer to chaos through the process of period-doubling. The corresponding complex regimes of motion are identified by a symbolic sequence.

\section{Bifurcation diagram}

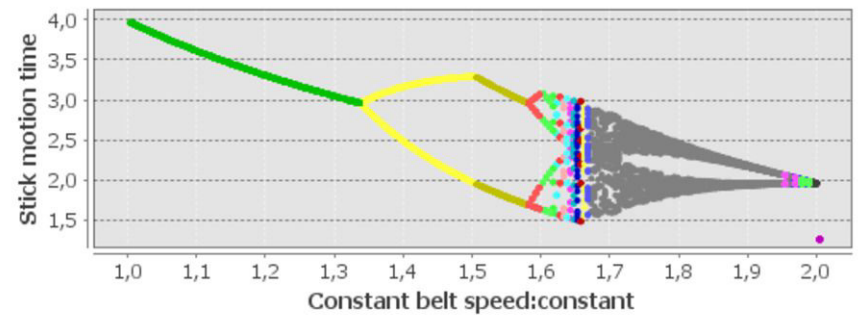

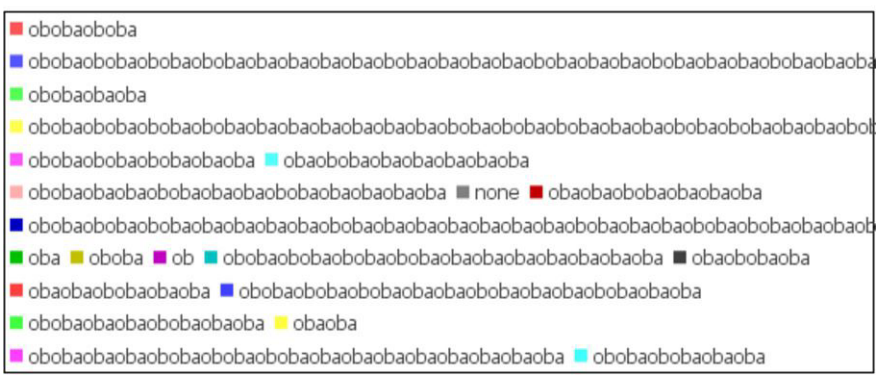

Fig. 4. Bifurcation diagram

This work was supported by a grant from the Government of the Russian Federation (contract No. 14.Y26.31.0031) 


\section{References}

1. A. Yu. Ishlinskiy, I. V. Kragelskiy, Zhurn. tehn. fiziki., E.14 4/5 (1944)

2. L. Ya. Kashenevskiy, Inzh.-fiz-zhurn., 47(1) (1984)

3. M. M. Vertyukov, S. V. Dobroslavskiy, R. F. Nagayev, Izv. AN SSSR. MTT, 1 (1990)

4. V. S. Metrikin, R. F. Nagayev, V. V. Stepanova, PMM, 60(5) (1996)

5. M. V. Zaytsev, V. S. Metrikin, Vestnik Nizhegorodskogo Universiteta Lobachevskogo, 3(1) (2012)

6. R. I. Leine, D. H. Van Campen, De Kraker, Nonlinear Dynamics, 16 (1999)

7. B. L. Van De Vrande, D. H. Van Campen, De Kraker, Nonlinear Dynamics, 19(2) (1999)

8. R. I. Leine, D. H. Van, Archive of Applied Mechanics, 72 (2002)

9. R. I. Leine, D. H. Van Campen, European Journal of Mechanics A/Solids, 25 (2006)

10. G. W. Luo, X. H. Lv, L. Ma, European Journal of Mechanics A Solids, 27 (2008)

11. M. M. Vetyukov, M. Yu. Platovskikh, Trudy Vserossiyskoy nauchno-metodicheskoy konferencii "Sovremennye problem mehaniki I eyo prepodavaniya v vuze". StPetersburg, Voyenno-kosmicheskaya akademiya Mozhayskigo (2015)

12. M. I. Feygin, Forced oscillations of systems with discontinuous nonlinearities (Nauka, Moscow, 1994)

13. Yu. I. Neymark, The point mapping method in the theory of nonlinear oscillations (Nauka, Moscow, 1972)

14. G. Shuster, Deterministic chaos (Mir, Moscow, 1988) 